\title{
Simulation Design of AOS Cross Layer Transmission
}

\author{
Bi Mingxue ${ }^{\mathrm{a}}$, Wang Hongxia ${ }^{\mathrm{b}}$ \\ ${ }^{a, b}$ Shenyang Ligong University, Shenyang, China \\ ${ }^{a}$ Automation Department, Nanjing University of Science and Technology, Nanjing, China
}

\begin{abstract}
In this paper, the AOS effective throughput rate is analyzed firstly. Then, in order to improve the effective throughput rate, a scheme of AOS cross layer transmission system is presented. In the proposed scheme, based on the space channel state, the dynamic optimization parameters of AOS Space Date Link Protocol sublayer and AOS Sync and Channel Coding sublayer are selected. Then the switch thresholds are presented. Finally, the effective throughput rate of the proposed AOS cross layer transmission system is simulated. Simulations show that the throughput rate can be optimized effectively.
\end{abstract}

Index Terms: AOS; cross layer transmission; effective throughput rate

(C) 2011 Published by MECS Publisher. Selection and/or peer review under responsibility of the Research Association of Modern Education and Computer Science

\section{Introduction}

AOS is used to manage and transfer various space mission data, such as the exploring and controlling data, the space communications data and other effectual loads data, over the space-to-ground, ground-to-space or space-to-space communication links. AOS needs to offer high speed and reliability for various space mission data and afford the Quality of Service for end to end transfer.

However, the time variety character of space link will limit the system throughput performance. So it is important to conquer the effect of space link (or physical channel) for improving the AOS effective throughput rate. The AOS throughput performance has been studied by literature [1] which discusses the impacts of frame length and the relevant solutions on the throughput. But the cross layer transmission scheme has not proposed. However, in wireless systems, cross layer techniques are generally used to enhance the throughput performance through adapting the different layers transmission parameters to the variations of the wireless channel. Nowadays, the concept of cross layer has been widely accepted. As a result, a large number of cross layer design proposals have appeared in the recent literature [2]-[4].

In this paper, an AOS cross layer transmission scheme is proposed to increase the AOS effective throughput rate based on intrinsic relations between the channel state, the throughput rate and each parameter of AOS different layers. To detail the principle and performance of the proposed scheme, the remainder of the paper is organized as follows: A brief explication of the AOS data transfers structure and cross layer structure is given in

* Corresponding author.

E-mail address: ${ }^{\text {bm }}$ 418@163.com 
section 2 and section 3 respectively. In section 4, AOS effective throughput rate formula of is introduced. Then the AOS cross layer system is designed in section 5, followed by concluding remarks drawn in Section 6 .

\section{AOS Data Transmission Structure}

The data transfers structure of AOS is layered as Fig. 1.

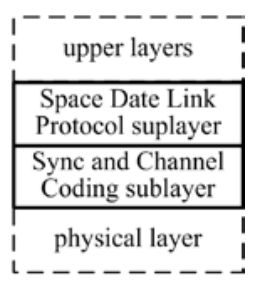

Fig 1. AOS data transfers structure

The AOS standard advises that the continuous different service data transferring from upper layers are partition to be fixed length transfer frames in Space Date Link Protocol (SDLC) sublayer. Then, these AOS transfer frames are coded and placed an Attached Sync Marker (ASM) in Sync and Channel Coding (SCC) sublayer, and send in physical layer. The traditional AOS layered structure is designed for the worst or the average space channel, and the communications in adjacent layers is invariable. It is unable to adapt the time-varying capacity and high bit error rate $(B E R)$ space channel. Consequently, increasing the throughput performance requires a cross layer joint design and optimization approach to dynamically adjusting the parameters of different layer.

\section{AOS Cross Layer Transmission Structure}

The structure of AOS cross layer transmission is shown in Fig. 2. Obtaining the channel Signal Noise Ratio $(S N R)$ from the estimator, controller decides the frame length of SDLP sublayer, and coding efficiency and frame sync parameters of SCC sublayer to maximize the AOS effective throughput rate.

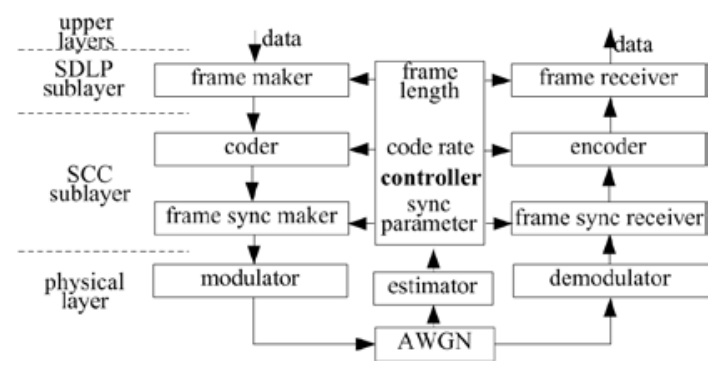

Fig 2. AOS cross layer structure

The AOS cross layer system is research with following presumptions and variable introductions:

- SNR of current channel can be check and feed back to the sender accurately.

- The frame queue in SDLP sublayer is saturated and the "bad" frames can be detect and drop reliably with CRC field.

- Teffective is the AOS effective throughput rate.

- Eup is the efficiency of upper layers. It relates to service types and PRI and AOS multiplexing. 
- Lframe is the length of frame.

- Lframe_c is the length of frame primary header and CRC field.

- Lframe_opt is the optimized length of frame.

- Lframe_ada is the adaptive length of frame.

- LFLM is the length of Frame Length Marker.

- Eframe is the efficiency of frame.

- Rcode is code rate.

- Esync is the efficiency of frame sync.

- LASM is the length of ASM.

- Rmodulation is modulation rate.

- Psuccess_sync is the successful probability of frame sync.

- FER is the frame error rate.

- Psuccess_frame is the successful probability of receiving frame.

- $\mathrm{R}$ is the symbol rate of physical channel.

\section{Analysis of AOS Effective Throughput Rate}

The effective throughput rate is the ratio of successfully received bit numbers to the total sending bit numbers. Based on the section 3, we have:

$$
T_{\text {effective }}=E_{\text {up }} E_{\text {frame }} R_{\text {code }} E_{\text {sync }} R_{\text {modulation }} P_{\text {success_sync }} P_{\text {success_frame }} R
$$

Where, $E_{\text {frame }}=\left(L_{\text {frame }}-L_{\text {frame_c }}\right) / L_{\text {frame }}, E_{\text {sync }}=L_{\text {frame }} /\left(L_{\text {frame }}+L_{\text {ASM }}\right), P_{\text {success_frame }}=1-F E R$.

Obviously, the parameters of each layer affect the AOS effective throughput rate. So combined optimization can enhance the effective throughput rate.

\section{Design of AOS Cross Layer Transmission}

\subsection{Design of Frame Length in SDLP sublayer}

If the effect of SCC sublayer is unconsidered, FER can be expressed as $F E R=1-(1-B E R)^{8 L_{\text {frame }}}$.

So, letting $\partial \ln T_{\text {effective }} / \partial L_{\text {frame }}=0$, we can get $L_{\text {frame_opt }}$ maximizing the throughput as

$L_{\text {frame_opt }}=\left(L_{\text {frame_c }}-L_{A S M}\right)+\sqrt{\frac{\left(L_{\text {frame_c }}+L_{A S M}\right)^{2}-\left(L_{\text {frame } \_}+L_{A S M}\right)}{8 \ln (1-b e r)}}$

From (2), we can obtain that $L_{\text {frame_opt }}$ is only determined by BER.

Based on the analysis above, we present frame length adaptive scheme. Before sending data, a pretreatment which chooses an appropriate frame length according to the channel states. The pretreatment can be performed by software, which needn't change the AOS architecture, so it is can be realized easily. Apparently, $L_{\text {frame_opt }}$ is continuous in (2). However, continuous frame length may increase the system complexity and cause performance instability. The discrete frame lengths are more suitable for the practical data transmission, and the frame length being an integer times of byte can be treated easily. As Table 1, the discrete frame lengths 128, 312 and 1024 bytes are considered and the Frame Length Marker (FLM) is defined. We define the FLM in frame primary header as Fig.3. So, $L_{F L M}=2$ bits. 
Table 1 Selection of Frame Length and FLM

\begin{tabular}{|l|l|c|}
\hline $\boldsymbol{B E R}$ & $\boldsymbol{L}_{\text {frame_ada }}($ bytes) & $\boldsymbol{F L M}$ \\
\hline$B E R \leq 6 \times 10^{-6}$ & 1024 & 00 \\
\hline $6 \times 10^{-6}<B E R \leq 2 \times 10^{-5}$ & 512 & 01 \\
\hline $2 \times 10^{-5}<B E R$ & 128 & 10 \\
\hline
\end{tabular}

\begin{tabular}{|c|c|c|c|c|c|c|c|}
\hline $\begin{array}{l}\text { master channel } \\
\text { ID }\end{array}$ & virtual & virtual & & Signa & aling fi & ield & frame \\
\hline 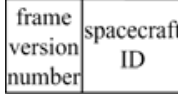 & $\begin{array}{c}\text { channel } \\
\text { ID }\end{array}$ & $\begin{array}{c}\text { channel } \\
\text { frame } \\
\text { count }\end{array}$ & $\begin{array}{c}\text { replay } \\
\text { flag }\end{array}$ & \begin{tabular}{|c|} 
VC frame \\
count usage \\
flag
\end{tabular} & FLM & $\begin{array}{c}\mathrm{VC} \text { frame count } \\
\text { cycle }\end{array}$ & $\begin{array}{c}\text { header } \\
\text { error } \\
\text { control }\end{array}$ \\
\hline
\end{tabular}

Fig 3. design of frame primary header

Letting $L_{\text {frame_c }}=12$ bytes, $L_{A S M}=8$ bytes, and $R=1$ symbols/s, $T_{\text {effective }}$ with $L_{\text {frame_opt }}$ and $L_{\text {frame_ada }}$ is shown in Fig. 4.When $B E R>10^{-4}$, the communications will be stopped because of too low $T_{\text {effective. }}$ Fig. 4 shows that $T_{\text {effective }}$ with $L_{\text {frame_ada }}$ is close to which with $L_{\text {frame_opt. }}$ So if channel states change, $T_{\text {effective }}$ can be improved effectively by the proposed adaptive frame length scheme compared with fixed frame length scheme, and the switch frequency of frame length is not as high as the optimal length scheme.

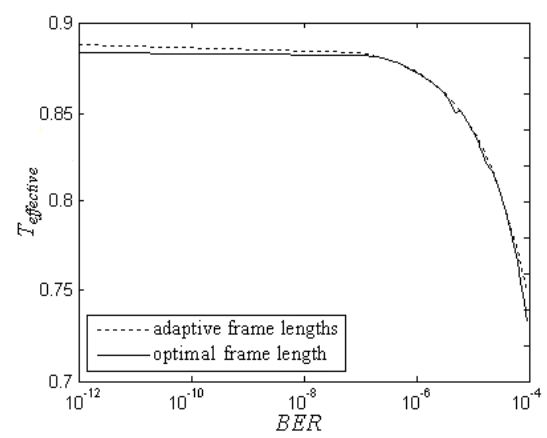

Fig 4. $T_{\text {effective }}$ with optimal and adaptive frame lengths

\subsection{Design of Code in SCC Sublayer}

Low Density Parity Check (LDPC) code is a class of block codes with near Shannon limit performance. It can provide very low error floors and very fast iterative convergence. These qualities make LDPC code a good fit for near Earth applications where very high data rates and high reliability are the driving requirements [5]. And LDPC code has been submitted to the CCSDS channel coding in the fall 2002 Houston meeting. In this paper, the linear block code is designated by $\left(L_{\text {frame }}, R_{\text {code }}\right)$. Fig. 5 shows the FER (solid) and the simulation FER (dashed) for the LDPC codes by BPSK modulation in physical layer. From left to right, these six codes have parameters $(128,1 / 2),(512,2 / 3)$ and $(1024,4 / 5)$. 


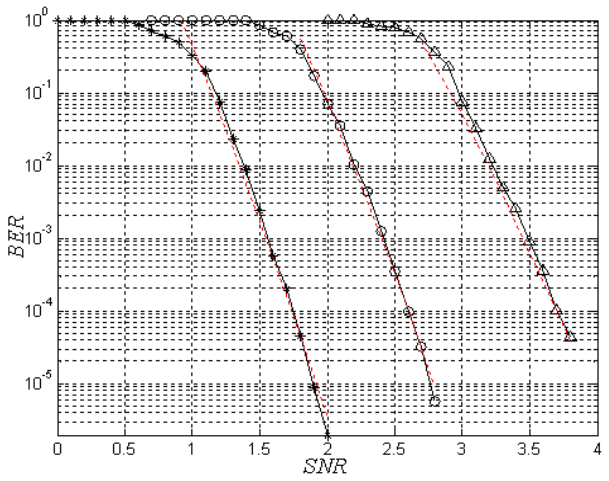

Fig 5. FER for the LDPC codes

The simulation FER can be implemented as (3), and the simulation parameters are shown as Table 2 [6, 7].

$$
F E R=p \exp (-q \cdot S N R)
$$

Table 2 the simulation parameters for FER

\begin{tabular}{|c|c|c|c|c|}
\hline model & $\boldsymbol{L}_{\text {frame ada }}$ & $\boldsymbol{R}_{\text {code }}$ & $\boldsymbol{p}$ & $\boldsymbol{q}$ \\
\hline 1 & 128 & $1 / 2$ & 109.56 & 21.55 \\
\hline 2 & 512 & $2 / 3$ & 10.12 & 2.23 \\
\hline 3 & 1024 & $4 / 5$ & 9.81 & 1.87 \\
\hline
\end{tabular}

\subsection{Design of frame sync in SCC Sublayer}

Frame sync is achieved by sync of an ASM associated with each LDPC Code block. The ASM is a bit pattern of CCSDS Recommended Standard [6], and it precedes the LDPC codeblock. Frame synchronizers should be set to expect a marker at a recurrence interval equal to the length of the ASM plus that of the LDPC Code block. All codes in the LDPC family use the 8 bytes ASM. Setting a limit to the frame sync error, the probability of false alarm $P_{F A}$ and the probability of false leak $P_{F L}$ are expressed as

$$
\begin{aligned}
& P_{F A}=\sum_{i=0}^{J} C_{8 L_{A S M}}^{i}(1 / 2)^{8 L_{A S M}} \\
& P_{F L}=1-(B E R)^{L_{F L M}} \sum_{i=0}^{J} C_{8 L_{A S M}}^{i}(1-B E R)^{8 L_{A S M}-i} B E R^{i}
\end{aligned}
$$

Where, $J$ is the maximal number of error bits in ASM. The relation of channel $S N R$ to BER are given as follows

$$
B E R=\operatorname{erfc}(\sqrt{S N R}) / 2
$$


If the value of $J$ is large enough, $P_{F A}$ can be ignored. So, $P_{\text {success_sync }}$ can be defined as

$$
P_{\text {sync }}=\frac{\left(1-P_{F L}\right)^{a}}{(1-B E R)^{L_{F L M}}}=\frac{\left(1-P_{F L}\right)^{a}}{(1-\operatorname{erfc}(\sqrt{S N R}) / 2)^{L_{F L M}}}
$$

Where $a$ is the times for checking the ASM successfully. $P_{\text {success_frame }}$ is given as

$$
P_{\text {success_frame }}=P_{\text {sync }}(1-p \exp (-q \cdot S N R))
$$

Setting $E_{\text {up }} R_{\text {modulation }} R=k, T_{\text {effective }}$ is given as

$$
\begin{aligned}
& T_{\text {effective }}=k\left(L_{\text {frame }}-L_{\text {frame_c }}\right)(1-p \exp (-q \cdot S N R))\left((\operatorname{erfc}(\sqrt{S N R}) / 2)^{L_{F L M}}\right. \\
& \frac{\left.R_{\text {code }} \sum_{i=0}^{J} C_{8 L_{A S M}}^{i}(1-\operatorname{erfc}(\sqrt{S N R}) / 2)^{8 L_{A S M}-i}(\operatorname{erfc}(\sqrt{S N R}) / 2)^{i}\right)^{a}}{\left(L_{F}\right.}
\end{aligned}
$$

\subsection{Design of switch thresholds of cross layer system}

The AOS cross layer system is simulated with following presumptions: $k=1, J=3 b i t s$ and other parameters as above paragraphs. The simulation of $T_{\text {effective }}$ with different models is shown in Fig.6.

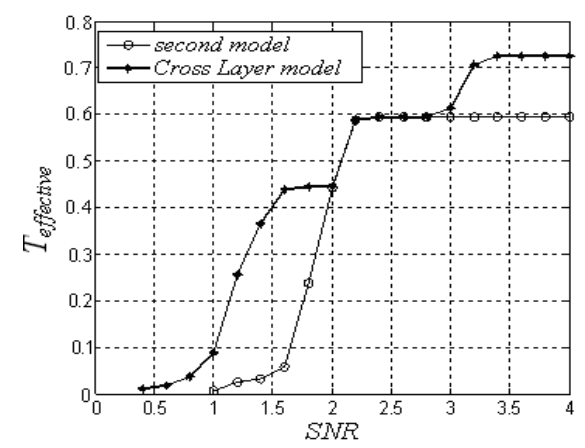

Fig 6. $T_{\text {effective }}$ with different models

From Fig.6 we can get the switch thresholds of SNR as table 3. Here the cross layer scheme is proposed to maximize throughput by suitably determining discrete adaptive frame length $L_{\text {frame_ada }}$ based on different ranges of $S N R$. When $S N R<1.5$, the communications will be stopped because of too low $T_{\text {effective }}$.

Table 3 the threshold of AOS cross layer system

\begin{tabular}{|c|c|}
\hline model & SNR threshold(dB) \\
\hline 1 & 1.80 \\
\hline 2 & 2.01 \\
\hline 3 & 2.98 \\
\hline
\end{tabular}


Fig. 6 shows the effective throughput rate of AOS cross layer scheme. So if channel SNR change, the throughput rate can be improved effectively by the proposed scheme compared with three fixed schemes, and the switch frequency of cross layer system is not high. The scheme is simple and need not change the frame length and code rate frequently, so the stability of the AOS and the reliability of data transmission can be guaranteed. So it is more suitable for practical data transmission.

\section{Conclusions}

An AOS cross layer scheme uses appropriate frame length, code rate and sync parameter for the throughput rate improvement is presented in this paper. First of all, a effective throughput rate formula of AOS has been proposed. From which we know that the effective throughput is mainly affected by frame length, coding and channel SNR. So a cross layer scheme controlling these parameters of different layers based on the ranges of $S N R$ is provided for maximizing the effective throughput rate, which needn't modify the AOS protocol too much. Simulation results show that compared with fixed transmission scheme, the proposed scheme can improve the AOS throughput rate effectively. Using the presented result, AOS protocol designers can verify and optimize their designs which provide a reference to the future application of space missions. In addition, this research does not consider the probability of modulation to AOS effective throughput rate. Further research is needed to support the performance analysis of more complicated communications.

\section{References}

[1] Bi Mingxue, Pan Chengsheng and Zhao Yuntao, Throughput Analysis and Frame Length Adaptive for AOS Packet Service, IEEE, 2009.

[2] Mihaela van der Schaar, Santhana Krishnamachari, Sunghyun Choi, et al.. Adaptive cross-layer protection strategies for robust scalable video transmission over 802.11 WLANS [J]. IEEE J. on Select., Area. Commun., 2009, 21(10): 1752-1763.

[3] Dai L, Letaief K B. Throughput maximization of adhoc wireless networks using adaptive ooperative diversity and truncated ARQ [DB/OL]. http:// hdl. handle. net/ 1783. 1/ 2252, 2007/ 20080921.

[4] G. Aniba and S. Aissa. Adaptive scheduling for MIMO wireless networks: Cross-layer approach and application to HSDPA. IEEE Trans. Wireless Commun., 6(1):259-268, Jan. 2007.

[5] Low Density Parity Check Codes for Use in Near_earth and Deep Space Applications [S]. Research and Development for Space Data System Standards, CCSDS 131.1-O-2 Orange Book, Issue 2. Washington, D.C.: CCSDS, September 2007.

[6] TM Synchronization and Channel Coding[S]. Recommendation for Space Data System Standards, CCSDS 131.0-B-1 Blue Book, Issue 1. Washington, D.C.: CCSDS, September 2003.

[7] AOS Space Data Link Protocol, CCSDS 732.0-B-2 Blue Book, Consultative Committee for Space Data System, July, 2006 